\title{
Signal probability effects on high-workload vigilance tasks
}

\author{
GERALD MATTHEWS \\ University of Dundee, Dundee, Scotland
}

\begin{abstract}
Signal probability is an important influence on vigilance. Typically, higher signal probability is associated with higher hit rate, lower response criterion, and lower response:signal ratio. However, signal probability effects on demanding, high-workload vigilance tasks have not been investigated. It is believed that attentional resources become depleted during performance of such tasks, leading to perceptual sensitivity decrements. Forty subjects performed high- (.35) and low- (.10) probability versions of a demanding vigilance task. Results differed in two important respects from those previously obtained with less demanding tasks. First, the decrement in perceptual sensitivity over time was greater for the high-probability task. Second, there were no effects of signal probability on response criterion. Subjective workload was higher for the high-probability task. Implications of the data for resource-depletion and expectancy theories of vigilance are discussed.
\end{abstract}

Signal probability has been widely recognized as one of the most important task factors influencing vigilance performance. Increases in signal probability tend to lead to increases in hit rate, the proportion of signals detected or correctly discriminated from nonsignals. In general, this finding supports expectancy theories of vigilance, which propose that hit rate improves with expectation of signal occurrence (see Davies \& Parasuraman, 1982). However, signal detection theory (SDT) analyses (see, e.g., Baddeley \& Colquhoun, 1969) have shown that probability is inversely related to response criterion (typically indexed by $\beta$ ), but has no effect on perceptual sensitivity (typically indexed by $d^{\prime}$ ).

There has been some debate over the observer's exact use of signal probability information. Williges (1973) has proposed that subjects use their knowledge of signal probability to set response criterion to an optimum value $\left(\beta_{\text {opt }}\right)$ that maximizes expected value of outcome. Suppose that signal probability, the proportion of events that constitutes signals, is designated by $p$. If the payoff matrix describing the values of different stimulus-response outcomes is symmetrical, $\beta_{\mathrm{opt}}$ is given by $(1-p) / p$ (Green $\&$ Swets, 1966). However, although this theory has had some success, subjects tend to be less extreme in their choice of criterion than the theory predicts (Davies \& Parasuraman, 1982). Craig (1987) has proposed that subjects typically adopt a response-biasing strategy of probability matching, attempting to equate total frequency of responses to the frequency of signals. Craig's (1978) re-

I am grateful to Andrew Griffiths for assistance in running this study. Correspondence should be addressed to G. Matthews, Department of Psychology, University of Dundee, Dundee DD1 4HN, Scotland (e-mail: g.matthews@dundee.ac.uk). view of vigilance studies suggests that the response frequency to signal frequency $(R: S)$ ratio is frequently larger than $l$ at the beginning of performance, perhaps due to incorrect expectancies. In these cases, $\mathrm{R}: \mathrm{S}$ ratio tends to decline toward 1 during the vigil. Craig's (1978) summary data suggest that $\mathrm{R}: \mathrm{S}$ ratio is initially higher when signal probability is low, perhaps because subjects are more liable to overestimate probability when signals are infrequent, as is often the case in vigilance studies. However, Kubovy (1977) has pointed out that conservative criterion placement in decision-making tasks may result from misconceptions concerning underlying probability distributions, rather than from response bias.

Temporal decline in hit rate is more commonly associated with increment in $\beta$ than with decrement in $d^{\prime}$ (Davies \& Parasuraman, 1982). However, vigilance tasks characterized by high information-processing demands or workload do reliably show sensitivity decrements (See, Howe, Warm, \& Dember, 1995). Davies and Parasuraman (1982) identified memory load and a high event rate $(>24 / \mathrm{min})$ as the critical factors necessary for sensitivity decrement. Such tasks may require a short-term memory matching process that tends to become fatigued over time (Parasuraman, 1979; Todkill \& Humphreys, 1994). Subsequent studies have suggested that high event rate and memory load are normally sufficient, but not necessary, for sensitivity decrement. For example, Neuchterlein, Parasuraman, and Jiang (1983) demonstrated that discrimination of rapidly presented single-digit targets shows a $d^{\prime}$ decrement when stimuli are perceptually degraded. In this instance, the decrement appears to be induced by the demands of processing poor-quality stimulus representations, rather than by short-term memory matching. See et al.'s (1995) meta-analysis of the sensi- 
tivity decrement suggests that the phenomenon is more widespread than originally suggested by Davies and Parasuraman (1982). Task difficulty (i.e., overall perceptual sensitivity) is associated with larger $d^{\prime}$ decrements, independent of the effects of high event rate and memory load. Effects of task factors on sensitivity decrement are consistent with an attentional resource model which states that various high task demands induce fatigue and progressively reduce attentional resource availability (Matthews, Davies, \& Holley, 1993).

Signal probability effects on demanding, high event rate vigilance tasks have been neglected. Expectancy theories (see, e.g., Williges, 1973) provide little explicit basis for predicting a moderating effect of task demands on probability effects. However, as Davies and Parasuraman (1982, p. 78) have indicated, expectancy theories involve assumptions about subjects' abilities to construct, remember, and modify a model of the statistical structure of the task, and these processes themselves may require attentional resources. If subjects' processing of probability information is impaired when task demands are high, we might expect weaker effects of signal probability on demanding tasks than on more "traditional," less demanding vigilance tasks. In addition, to the extent that increased signal probability leads to increased response rate, we might expect that high signal probability would lead to greater mental fatigue and resource depletion, and hence to greater temporal decrement in perceptual sensitivity.

The goal of the present experiment was to test the effects of two signal probabilities (.10 and .35) on a high event rate task in which the subject is required to discriminate degraded single-digit targets (Matthews, Davies, \& Lees, 1990; Neuchterlein et al., 1983). The task shows reliable sensitivity decrements at signal probabilities of .25 (Matthews, Davies, \& Lees, 1990) and .15 (Matthews et al., 1993), together with the criterion increments found with most vigilance tasks. It also appears to be subjectively fatiguing, with self-report energetic arousal decreasing during the period of work (Matthews, Jones, \& Chamberlain, 1990). Assuming symmetry of the payoff matrix, Williges's (1973) expectancy theory predicts that $\beta$ should tend toward optimal values of 9 on the lowprobability task and 1.86 on the high-probability task. It might be expected that rate of change of $\beta$ would be greater for the task version for which $\beta$ was initially most discrepant from the optimum. The probability-matching hypothesis predicts $\mathrm{R}: \mathrm{S}$ ratios approaching 1.0 on both tasks, with a larger initial R:S ratio and a stronger temporal decline in R:S ratio on the low-probability task. The resource-depletion hypothesis predicts a larger decrement in perceptual sensitivity on the high-probability task. Subjective workload was also measured, using the NASA-TLX measure (Hart \& Staveland, 1988), to test whether signal probability influences subjects' appraisals of the task. The resource-depletion hypothesis predicts higher workload for the high-probability task. Previous studies have shown that workload for high event rate vigilance tasks is often comparable to that of demanding tasks such as mental arithmetic (Deaton \& Parasuraman, 1993).

\section{METHOD}

\section{Subjects \\ Twenty male and 20 female undergraduate students, between 18 and 22 years old, participated in the study.}

\section{Design}

Data were analyzed within a $2 \times 3$ (signal probability $\times$ task period) factorial design, with repeated measures on both factors.

\section{Tasks}

The vigilance task was similar to that used by Matthews et al. (Matthews, Davies, \& Lees, 1990; Matthews et al., 1993). Stimuli were presented on a monochrome VDU by a BBC Model B microcomputer. Each stimulus was a single digit, displayed for $40 \mathrm{msec}$ as part of a $32 \times 32$ block of pixels in the center of the screen. All stimuli were visually degraded so that, for each stimulus, $30 \%$ of the pixels were reversed in polarity; some of the pixels of the white digit became black, and some of the pixels of the black background became white. The specific choice of pixels to be reversed on each trial was determined by random selection of 1 out of 10 masking patterns used throughout the task. Stimuli were viewed from a distance of $2.2 \mathrm{~m}$ and subtended an angle of approximately $0.47^{\circ}$ horizontally and $0.57^{\circ}$ vertically. Stimuli were presented at an event rate of $1 / \mathrm{sec}$. Subjects were instructed to press a key on a response box when they saw the digit 0 . Other digits from 1 through 9 constituted distractors and were presented with equal probability. Subjects performed the task twice, at two levels of signal probability. Each task version lasted for $9 \mathrm{~min}$. In the low-probability condition, subjects were presented with 540 stimuli, of which $54(10 \%)$ were targets. In the high-probability condition, 189 out of 540 stimuli ( $35 \%$ ) were targets. Data were analyzed across three successive time blocks of 180 stimuli. The $d^{\prime}$ and $\beta$ values were calculated from standard formulae (Davies \& Parasuraman, 1982, pp. 47 and 50). $\beta$ values were transformed to natural logs.

\section{Procedure}

All subjects performed both vigilance tasks, with the order of performance counterbalanced across the sample. For each task, subjects began by viewing a sequence of 30 example stimuli, with target dig. its indicated. This was followed by a practice task with 120 stimuli.

Table 1

Effects of Signal Probability and Task Period on Various Performance Measures

\begin{tabular}{|c|c|c|c|c|c|c|c|c|c|c|c|c|}
\hline & \multicolumn{6}{|c|}{ Low Probability } & \multicolumn{6}{|c|}{ High Probability } \\
\hline & \multicolumn{2}{|c|}{ Period 1} & \multicolumn{2}{|c|}{ Period 2} & \multicolumn{2}{|c|}{ Period 3} & \multicolumn{2}{|c|}{ Period 1} & \multicolumn{2}{|c|}{ Period 2} & \multicolumn{2}{|c|}{ Period 3} \\
\hline & $M$ & $S D$ & $M$ & $S D$ & $M$ & $S D$ & $M$ & $S D$ & $M$ & $S D$ & $M$ & $S D$ \\
\hline Correct detection rate $(\%)$ & 95.8 & 7.3 & 94.8 & 8.8 & 93.1 & 12.2 & 96.3 & 5.6 & 92.6 & 9.9 & 89.6 & 12.6 \\
\hline False positive rate $(\%)$ & 3.75 & 3.38 & 3.93 & 2.67 & 3.20 & 2.48 & 3.00 & 2.32 & 5.27 & 2.72 & 4.05 & 2.87 \\
\hline$d^{\prime}$ & 4.15 & 0.77 & 4.03 & 0.67 & 3.99 & 0.88 & 4.07 & 0.56 & 3.50 & 0.74 & 3.48 & 0.76 \\
\hline $\log \beta$ & -0.08 & 1.26 & -0.07 & 1.52 & 0.33 & 1.15 & -0.08 & 1.25 & -0.20 & 1.19 & 0.40 & 1.33 \\
\hline $\mathrm{R}: \mathrm{S}$ ratio & 1.17 & 0.19 & 1.17 & 0.20 & 1.11 & 0.16 & 1.01 & 0.07 & 1.01 & 0.11 & 0.96 & 0.14 \\
\hline
\end{tabular}

Note-R:S, response frequency to signal frequency. 
Signal probability for both example and practice stimuli was the same as in the main 9-min task, which followed practice. No feedback was given during either practice or the main task run. After completion of the main task, subjects completed the NASA-TLX (Hart \& Staveland, 1988). Subjects provided ratings on a $0-100$ scale of six aspects of workload (mental, physical, and temporal demand; performance; effort; and frustration), and completed a series of paired comparisons of the importance of each aspect to overall workload (see Hart \& Staveland for a procedure for calculating an overall weighted workload measure from these data).

\section{RESULTS}

Means and standard deviations for the dependent measures are given in Table 1. The main effect of probability $(d f=1,39)$ was significant at $p<.01$ for false positive rate $(F=40.7), d^{\prime}(F=23.5)$, and $\mathrm{R}: \mathrm{S}$ ratio $(F=$ $53.1)$. The main effect of period $(d f=2,78)$ was significant at $p<.01$ for all five dependent measures: hit rate $(F=11.3)$, false positive rate $(F=14.7), d^{\prime}(F=13.6)$, $\log \beta(F=7.1)$, and $\mathrm{R}: \mathrm{S}$ ratio $(F=8.7)$. The probability $\times$ period interaction $(d f=2,78)$ was significant at $p<.05$ for $d^{\prime}(F=4.8)$ and at $p<.01$ for false positive rate $(F=4.8)$. Mean $d^{\prime}$ values on the two tasks were similar in the first task period, but there was a steeper decrement in $d^{\prime}$ in subsequent periods on the high-probability task. Separate analyses of the two tasks showed that the effect of period on $d^{\prime}$ was nonsignificant on the lowprobability task $[F(2,78)=0.8]$, but significant on the high-probability task $[F(2,78)=23.3, p<.01]$. Mean $d^{\prime}$ did not differ significantly across the two tasks in Period 1 $[t(39)=0.80]$, but intertask differences were significant in Period $2[t(39)=4.1, p<.01]$ and in Period $3[t(39)=$ $3.8, p<.01]$. Faster deterioration of performance in the high-probability condition was more evident for false positives than for hits. The probability $\times$ period interaction was significant for false positives, but not for hits, although the $F$ of 2.9 for the interaction was close to significance $(.05<p<.10)$ for this measure.

$\log \beta$ tended to increase over time, but probability had no significant effects on response criterion. In Period 3 , the mean value of $\log \beta(0.33)$ approached the optimum value of 0.62 (i.e., $\log 1.86$ ) on the high-probability task, but Period $3 \log \beta$ on the low-probability task $(0.40)$ was considerably less than the optimum of 2.20 (i.e., $\log 9$ ) for this task version. However, measures of central tendency may be misleading in that individual subjects showed considerable variation in $\beta$ and in change in $\beta$ during the period of work. Few subjects were close to $\beta_{\text {opt }}$ on either task in any task period. As a check on changes in $\beta$, subjects were categorized by whether $\beta$ became closer to $\beta_{\text {opt }}$ across Periods 1 and 3 , whether $\beta$ remained at the same value, or whether $\beta$ became more distant to $\beta_{\text {opt. }}$ On the low-probability task, $\beta$ became closer to the optimum in 22 subjects, stayed the same in 6 , and became further from the optimum in 12 . On the high-probability task, $\beta$ became closer to the optimum in 27 subjects, stayed the same in 1 , and became further from the optimum in 12. A substantial minority of subjects failed to show the general trend for $\beta$ values to become closer to the optimum value, and differences in the direction of change across the two tasks appear to be minor.

Table 1 shows that R:S ratio was higher on the lowprobability task than on the high-probability task. R:S ratio was also higher in the first two task periods than in the third period, although the probability $\times$ period interaction was nonsignificant. Mean R:S ratio was close to 1.0 in all conditions, but, as in the case of $\beta$, there was some individual variation in scores, shown in Figure 1 for task Periods 1 and 3. At all time periods, more subjects showed approximate probability matching on the high-probability task than on the low-probability task, with an R:S ratio between 0.96 and 1.05. Despite the change over time in mean $\mathrm{R}: \mathrm{S}$ ratio, there was little change in the proportions of subjects showing near-exact probability matching during the run. On the high-probability task, there was an increase in the third period in the number of subjects responding less than probability matching would require.

Mean overall workload values were $57.1(S D=13.6)$ for the low-probability task and $61.5(S D=13.9)$ for the high-probability task. These means differ significantly $[t(39)=2.2, p<.05]$. The only significant difference obtained in comparisons of the six workload ratings was that for mental demand. Mean rating was 48.9 ( $S D=$ $21.0)$ for the low-probability task and $56.1(S D=21.9)$ for the high-probability task $[t(39)=3.2, p<.01]$.

\section{DISCUSSION}

Signal probability effects on high event rate sustained attention may be rather different from those on more "traditional," low event rate vigilance tasks in two major respects. First, signal probability influenced the extent of the $d^{\prime}$ decrement, although the two tasks were matched for $d^{\prime}$ in the first task period. Sensitivity declined significantly over time only in the high-probability condition, implying that high signal probability may increase the likelihood that the $d^{\prime}$ decrements are found with tasks using degraded stimuli. Neuchterlein et al.'s (1983) original demonstration of the effect also used a fairly high signal probability $(.25)$. It is unlikely that there is any fixed threshold probability above which decrements are found; See et al.'s (1995) meta-analysis suggests that magnitude of sensitivity decrement is a complex function of a variety of task parameters. Second, signal probability failed to influence $\beta$, although optimum $\beta$ values for the two probability conditions differed considerably. It should be noted that $d^{\prime}$ values, ranging from 3.48 to 4.15 , were unusually high for vigilance tasks, so results may not generalize to tasks of lower $d^{\prime}$ values. However, characteristic vigilance findings are often evident in tasks with $d^{\prime}$ values comparable to those of the present tasks (see, e.g., Deaton \& Parasuraman, 1993).

The resource-depletion hypothesis successfully predicted the greater sensitivity decrement in the high-probability condition. The prediction of greater subjective workload for the high-probability task was also confirmed. In general, increased probability appears to be associated with increased vulnerability to mental fatigue and reduced resource availability during the period of work. More detailed explanation requires further consideration of the processing supporting performance. Todkill and Humphreys (1994) have described two qualitatively different processing strategies used in discrimination tasks: short-term memory matching, which involves detection of stimulus change across successive trials, and long-term comparison, which requires the stimulus to be compared with a standardized representation 
(a)

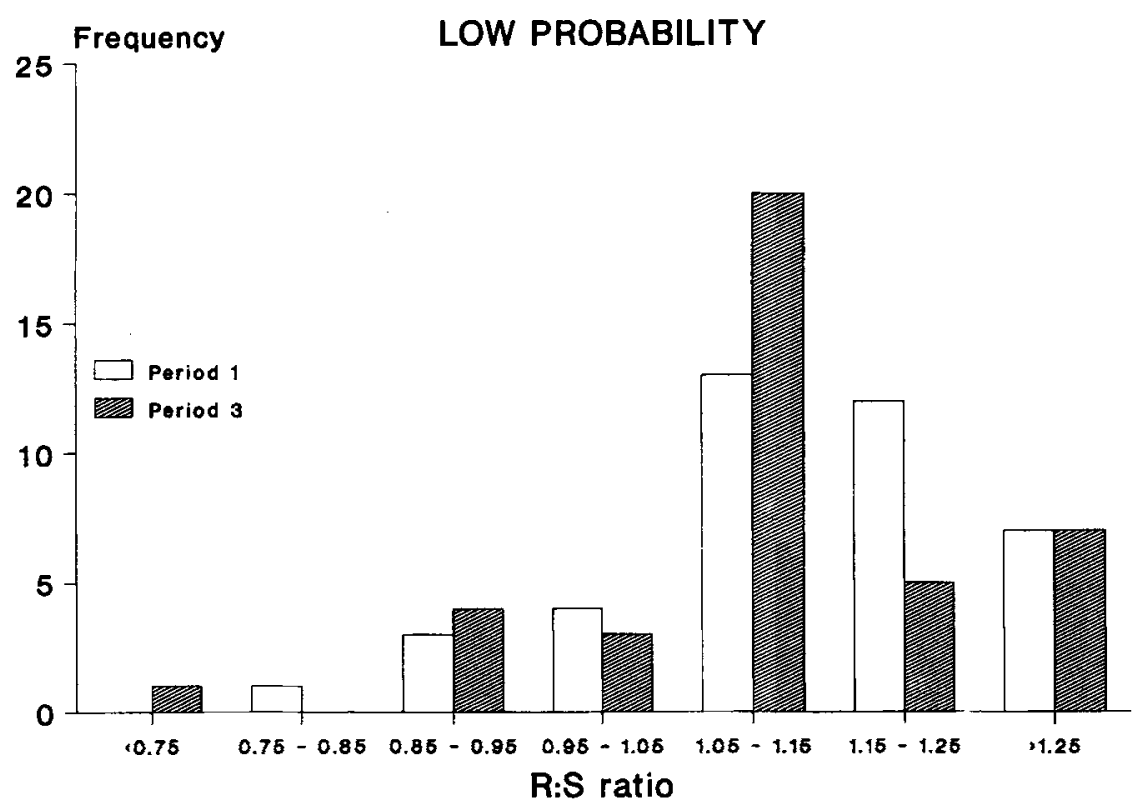

(b)

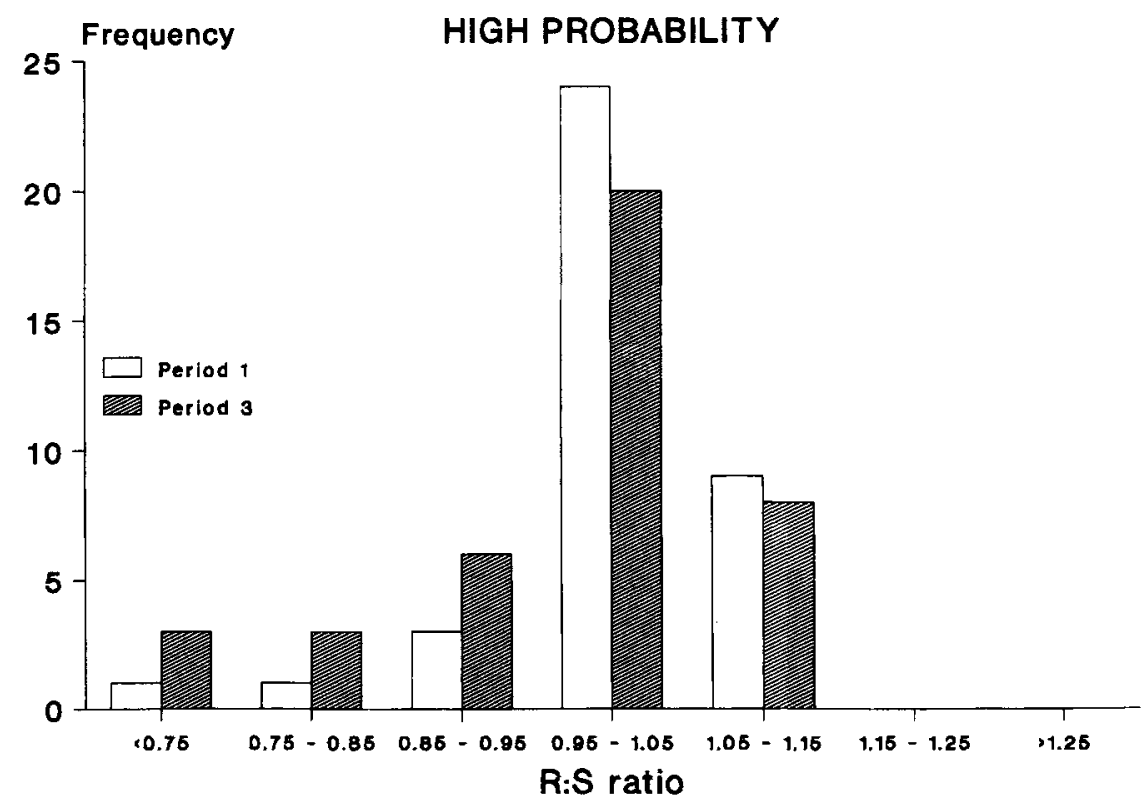

Figure 1. Distributions of the response frequency to signal frequency (R:S) ratio in task Periods 1 and 3, for (a) low-probability and (b) high-probability tasks. The ordinate is the number of subjects in each $R: S$ ratio band.

of the target stimulus in long-term memory. Short-term memory matching would not be effective for digit discrimination and it is plausible that long-term comparison is used. If so, fatigue effects might be associated with several distinct processes: initial encoding of the digit, deciding whether the stimulus matches the memory representation, and selecting and executing the response. Processes additional to shortterm memory comparison may contribute to mental fatigue and resource depletion.
One possibility is that stimulus encoding is the source of fatigue. Encoding the target digit, zero, might simply happen to be more effortful than encoding the other digits, so that fatigue increases with the proportion of zeros. Contrary to this hypothesis, choice of target digit is not critical; Matthews et al. (1993) showed a sensitivity decrement using "9" as the target. Also, sensitivity of target discrimination does not seem to be a direct function of encoding difficulty. Matthews et al. (1993) showed that neither overall sensitivity nor sensitivity 
decrement was significantly correlated with the person's ability to report masked digits, with no time pressure. Alternatively, selecting or executing the response may itself be fatiguing, and the probability effect derives from the increased response rate. If so, the most direct consequence should be a drop in response rate. In fact, as Table 1 shows, the decline in $d^{\prime}$ on the high-probability task precedes the decline in R:S ratio, $d^{\prime}$ drops primarily between Periods 1 and 2, and R:S ratio drops between Periods 2 and 3 .

The final possibility is that the decision process itself is fatiguing when it is difficult to judge whether or not a stimulus is a target or nontarget. In terms of SDT (Macmillan \& Creelman, 1991), the stimulus is associated with a certain likelihood ratio representing the odds in favor of the presence of a signal. Decision may be fatiguing when this stimulus likelihood ratio is close to the criterion value $(\beta)$. In the present study, $\beta$ values were initially close to 1 , implying a neutral criterion equidistant from the peaks of noise and signal distributions. However, some of the distractor digits are easier to discriminate from the target than others, so it is likely that different distractors are associated with noise distributions of differing means. Relatively easy distractors may typically generate a small stimulus likelihood ratio, considerably below the criterion value, and provide some respite from the fatiguing effects of relatively difficult distractors and targets, effectively reducing the event rate of the task. Increasing the signal probability replaces both easy and difficult distractors with targets. Hence, there is an increase in the total frequency of relatively difficult stimuli presented in a given time interval (i.e., targets plus difficult distractors), and so the task becomes more fatiguing, generating sensitivity decrement and increased subjective workload. Thus, it may be possible to manipulate workload and size of $d^{\prime}$ decrement on the degraded digits task by systematically manipulating discriminability of the various distractors from the target.

The data on criterion setting provide only limited support for the expectancy theories of Williges (1973) and Craig (1987). There was only a weak tendency for $\beta$ values to converge toward $\beta_{\text {opt }}$ values during the period of work, though it is conceivable that $\beta$ values would move closer to $\beta_{\text {opt }}$ during a more extended vigil. Although the normal temporal increment in $\beta$ was obtained, signal probability had no effect on either initial level of $\beta$ or temporal change in $\beta$. Because both tasks are fairly demanding, as shown by the workload data, the insensitivity of $\beta$ to signal probability may result from a lack of resources available for processing probability information. Probability matching may require fewer attentional resources than computation of optima $\beta$ values, and so may be the favored strategy for controlling readiness to respond on demanding tasks.

Although $\beta$ and R:S ratio were negatively correlated, as expected, $\mathrm{R}: \mathrm{S}$ ratio was more sensitive to signal probability than was $\beta$. The higher initial $\mathrm{R}: \mathrm{S}$ ratio in the low-probability condition is consistent with Craig's $(1978,1987)$ results. On the low-probability task, the mean shifted toward 1.0, as expected, but the modal tendency was toward an R:S ratio between 1.05 and 1.15 . Near-exact probability matching was more pronounced on the high-probability task, but it is difficult for the probability-matching hypothesis to explain why $\mathrm{R}: \mathrm{S}$ ratio should decline significantly on the high-probability task when mean R:S ratio initially was close to 1.0 . The decline in the mean appeared to be associated with increasing numbers of subjects for whom $R: S$ ratio was less than 1.0. Craig (1978) found a nonsignificant decline in R:S ratio in studies in which R:S ratio was initially less than 1.0 , but as Davies and Parasuraman (1982) have pointed out, the probability-matching hypothesis predicts a temporal increment in $\mathrm{R}: \mathrm{S}$ ratio under these circumstances. The probability-matching hypothesis is consistent with the general trend toward near-unity $R: S$ ratios in most subjects, but it does not appear to provide a full explanation for temporal change in $\mathrm{R}: \mathrm{S}$ ratio.

\section{REFERENCES}

BAddeley, A. D., \& Colquhoun, W. P. (1969). Signal probability and vigilance: A reappraisal of the "signal rate" effect. British Journal of Psychology, 60, 165-178.

CraIG, A. (1978). Is the vigilance decrement simply a response adjustment towards probability matching? Human Factors, 20, 441446.

CRAIG, A. (1987). Signal detection theory and probability matching apply to vigilance. Human Factors, 29, 645-652.

Davies, D. R., \& Parasuraman, R. (1982). The psychology of vigilance. London: Academic Press.

Deaton, J. E., \& Parasuraman, R. (1993). Sensory and cognitive vigilance: Effects of age on performance and subjective workload. Human Performance, 6, 71-97.

GREEN, D. M., \& SWETS, J. A. (1966). Signal detection theory and psychophysics. New York: Wiley.

Hart, S. G., \& STaveland, L. E. (1988). Development of a multidimensional workload rating scale: Results of empirical and theoretical reseach. In P. A. Hancock \& N. Meshkati (Eds.), Human mental workload (pp. 139-183). Amsterdam: Elsevier, North-Holland.

Kubovy, M. (1977). A possible basis for conservatism in signal detection and probabilistic categorization tasks. Perception \& Psychophysics, 22, 277-281.

Macmillan, N. A., \& Creelman, C. D. (1991). Detection theory: A user's guide. Cambridge: Cambridge University Press.

Matthews, G., Davies, D. R., \& Holley, P. J. (1993). Cognitive predictors of vigilance. Human Factors, 35, 3-24.

Matthews, G., Davies, D. R., \& LeEs, J. L. (1990). Arousal, extraversion, and individual differences in resource availability. Journal of Personality \& Social Psychology, 59, 150-168.

Matthews, G., Jones, D. M., \& Chamberlain, A. G. (1990). Refining the measurement of mood: The UWIST Mood Adjective Checklist. British Journal of Psychology, 81, 17-42.

Neuchterlein, K., Parasuraman, R., \& Jiang, Q. (1983). Visual vigilance: Image degradation produces rapid sensitivity decrement over time. Science, 220, 327-329.

Parasuraman, R. (1979). Memory load and event rate control sensitivity decrements in sustained attention. Science, 205, 924-927.

SeE, J. E., Howe, S. R., WARm, J. S., \& Dember, W. N. (1995). Metaanalysis of the sensitivity decrement in vigilance. Psychological Bulletin, 117, 230-249.

Todkill, A. L., \& Humphreys, M. S. (1994). Stimulus comparison strategies and task demands in successive discrimination. Quarterly Journal of Experimental Psychology, 47A, 761-780.

WILLIGES, R. C. (1973) Manipulating the response criterion in visual monitoring. Human Factors, 15, 179-185.

(Manuscript received November 1, 1994; revision accepted for publication January 24, 1996.) 